\begin{tabular}{|l|l|l|l|l|l|}
\hline MUNIBE Antropologia-Arkeologia & $n^{\circ} 65$ & $239-247$ & DONOSTIA & 2014 & ISSN 1132-2217 • eISSN 2172-4555 \\
\hline
\end{tabular}

\title{
Cultura material y fuentes escritas en los estudios arqueológicos de las pesquerías vascas de Canadá (siglos XVI al XVIII), una valoración crítica sobre su interacción
}

\author{
Material Culture and Documentary Sources in Archaeological \\ Studies of Basque Fisheries in Canada (16 th to $18^{\text {th }}$ centuries), \\ a critical assessment on their interaction
}

KEY WORDS: Historical archaeology, basque whalers, fisheries, pottery

PALABRAS CLAVES: Arqueología histórica, balleneros vascos, pesquerías, cerámica

GAKO-HITZAK: Arkeologia historikoa, euskal baleontziak, arrantza tokiak, zeramika

Sergio ESCRIBANO-RUIZ ${ }^{(1)}$

\begin{abstract}
The text we are presenting aims to be a brief reflection on how historical knowledge is produced, an old issue we are taking up again due to the study of the pottery consumed by Basque fishermen in Canada from the $16^{\text {th }}$ to $18^{\text {th }}$ centuries. We argue that interaction between written sources and material evidence is as necessary as it is dangerous. Therefore, this epistemological challenge must be undertaken together with a critical reasoning process in order to build more contingent and less biased discourses. This reflection will be illustrated with a case study based on analyzing the identity of the Basques fishermen who went to Canada. It will be argued that the mainstream view is based on the written sources and that material culture is offering new interpretative frameworks.

It will be shown that the role of Biscayan sailors and merchants, and the importance of the Province of Araba in the logistics of the voyages and the trade in whale blubber, have traditionally been underestimated. It will be argued that this is directly connected with the study of archives from Gipuzkoa, and the lack of systematic studies of documents from Biscay and Araba in this matter. All these provincial characteristics need to be deconstructed to be able to characterise the Basque presence in Canada. It will be shown how the problems described above can be solved by the archaeological record and particularly by the pottery assemblage. Finally, the nature of written and material sources will be analysed, advocating their joint study, always accompanied by a critical interpretative process.
\end{abstract}

\section{RESUMEN}

Esta breve aportación es una reflexión sobre un viejo tema, la forma en las que se construye el conocimiento histórico; y ha sido inducida por un motivo muy concreto, el estudio de la cerámica consumida por los pescadores vascos en tierras canadienses entre los siglos XVI y XVIII. A lo largo del trabajo se argumentará que la interacción entre fuentes escritas y materiales es tan necesaria como peligrosa y que debe estar acompañada siempre de un proceso de razonamiento crítico que permita construir discursos más contingentes y menos sesgados. Todo ello será ilustrado mediante un estudio de caso centrado en el análisis de la identidad de los marinos vascos que se aventuraron en las pesquerías de Canadá. Se argumentará que la visión dominante se basa sobre todo en la documentación escrita y que la cultura material está proponiendo nuevos marcos interpretativos.

\section{LABURPENA}

Aurkezten dugun lan hau gai zahar baten inguruko hausnarketa labur bat baino ez da, ezagutza historikoaren sorkuntzaren gainekoa hain zuzen. Gogoetak, euskal arrantzaleek Kanadan XVI eta XVIII. menden artean erabili zituzten zeramikaren ikerketan du sorrera. Testuan zehar iturri idatzi eta materialen arteko elkarlana beharrezkoa izateaz gain arriskutsua ere badela defendatuko dugu. Horregatik, eta iraganaren eraikitzea zehatzagoa eta fidelagoa izan dadin, honelako ariketa epistemologikoak pentsamendu kritiko baten bidez eramanak izan behar direla uste dugu. Arrazonamendu guzti hau, Kanadara jo zuten arrantzaleen izaeraren ikerketan oinarritzen den ikerketa konkretu baten bidez bideratzen da. Orain arteko ikuspegi nagusia iturri idatzietan oinarritzen dela eta kultura materialak bestelako aukeren berri ematen duela ere adieraziko dugu.

(1) Grupo de Investigación en Patrimonio Construido, GPAC (UPV/EHU) Departamento de Geografía, Prehistoria y Arqueología. C/ Tomas y Valiente s.n. 01006. Vitoria-Gasteiz sergio.escribanor@ehu.es 


\section{1.- LA IMPLEMENTACIÓN DE LAS FUENTES HIS- TÓRICAS, UNA PELIGROSA NECESIDAD}

La tradición epistemológica europea ha fomentado desde hace tiempo el empleo de diferentes evidencias en la construcción de la Historia. Sin embargo, la interacción entre fuentes diversas y su integración en un mismo discurso no han sido estrategias tan promovidas. Ambas circunstancias han concurrido en el desarrollo de la arqueología, que siempre ha sido considerada una disciplina excepcional para trabajar en los periodos carentes de fuentes escritas, pero que ha estado bajo sospecha en el estudio de las sociedades escritoras. Alejada de un enfoque integrador e interdiciplinar de la Historia, ha sido utilizada para llenar los vacíos del conocimiento histórico y descartada ante la existencia de otras fuentes de evidencia. Y ha sido así hasta tal punto que, en la actualidad, la arqueología de época moderna, un periodo que goza de un buen repertorio de documentos escritos, se encuentra en el mismo estado de desarrollo que a finales del siglo XX, cuando el estado de la Arqueología Postmedieval en España se resumió desde su indefinición como disciplina (AMORES, 1997: 66).

En estas líneas defenderemos que cada fuente tiene una potencialidad hermenéutica concreta que aportar en la reconstrucción del pasado y que considerarlas de forma conjunta en un mismo proceso interpretativo, proporciona resultados inalcanzables desde perspectivas epistemológicas unilineales. Este es el enfoque con el que se está trabajando de forma ejemplar especialmente en el marco de la Arqueología Histórica de filiación americana, que ve en la multiplicidad de fuentes un escenario epistemológico privilegiado más que un motivo de exclusión disciplinar. Numerosas obras colectivas y manuales ofrecen ricos y novedosos ejemplos de esta forma de entender la reconstrucción del pasado cercano (entre muchos otros: FUNARI, HALL Y JONES, 1999; ORSER, 2002; HICKS, BEAUDRY, 2006; MAJEWSKI, GAIMSTER, 2009).

Alentada por las contradicciones entre las palabras y las cosas, la historiografía americana ha producido interesantes reflexiones sobre la interacción entre fuentes escritas y arqueológicas. En la exploración de esas contradicciones, han trabajado con conceptos como transdisciplinaridad, entendida como unión coyuntural ante problemas concretos en el que la solución está por encima de cualquier disciplina particular; o polifonía, en alusión al sonido propio de cada una de las distintas voces que constituyen la obra llamada Historia (JOYCE, 2006). Empeñados en dar voz a quienes no tienen un legado literario, han reclamado la necesidad de reflexionar críticamente sobre la causalidad de las fuentes empleadas en el estudio del pasado y sobre sus problemas de representatividad. Pero han demostrado también la necesidad de implementar las distintas fuentes históricas, aunque respetando siempre sus respectivas características hermenéuticas

Estas líneas son una invitación a superar el viejo paradigma histórico europeo, a tratar de construir una His- toria transdisciplinar e inclusiva capaz de incorporar el discurso de todas las fuentes disponibles. Sólo así podrá representar todas las voces que configuraron nuestro pasado, también aquellas menos recordadas, por haber sido silenciadas o más silenciosas.

\section{CANADÁ ENTRE LOS SIGLOS XVI Y XVIII, UN CRISOL DE IDENTIDADES}

El ejemplo escogido para tratar de demostrar la necesidad de confrontación y complementación de las fuentes históricas, especialmente en los casos de contacto cultural, es la actividad de los pescadores vascos en el extremo oriental de Canadá durante los dos primeros siglos de expansión colonial europea en América. En este escenario, a diferencia de lo sucedido en el la mayor parte de América, la interacción cultural entre nativos y colonizadores fue extremadamente compleja; especialmente porque las tierras y las costas canadienses eran una reserva económica internacional poblada por diferentes pueblos indios y esquimales, de la que se aprovecharon de forma simultánea numerosos pueblos europeos hasta el siglo XVIII, momento en el que su explotación fue monopolizada por las coronas de Francia e Inglaterra. Pero esa interacción también fue muy compleja entre los propios vascos, ya que por un lado se encontraban los súbditos de la corona francesa, los habitantes de Lapurdi (o vasco-continentales), y por el otro los vasallos de la corona castellana, habitantes de Araba, Bizkaia y Gipuzkoa (o vasco-peninsulares).

Nuestro discurso comenzará analizando la forma en las que se ha medido la presencia vasco-peninsular en Canadá a partir de diferentes evidencias. Después, nos detendremos en un reciente episodio historiográfico que abre nuevas vías de interpretación de la identidad de la empresa pesquera ultramarina del País Vasco peninsular y de sus relaciones con las sociedades locales. Trataremos de valorar finalmente el potencial de la cerámica como medio para diferenciar identidades culturales. Creemos, al igual que otros autores (WHITE, BEAUDRY, 2009; MAJEWSKI, SCHIFFER, 2009; BARKER, MAJEWSKI, 2006), que la cerámica puede ser una herramienta muy útil a la hora de estudiar los contactos culturales entre los pescadores vascos y las diferentes poblaciones locales, pero también dentro de cada uno de estos grupos. Nos centraremos especialmente en analizar la "identidad utilitaria" de los pescadores vascos, la relación distorsionada que existe entre las producciones cerámicas conservadas hoy y la identidad de las personas que las consumieron; tarea que creemos podría desarrollarse con éxito en este caso con la implementación de diversas fuentes materiales y literarias.

\section{LA PRESENCIA VASCA EN CANADÁ}

\subsection{Síntesis historiográfica}

No pretendemos ofrecer una visión exhaustiva de la historiografía relativa a la pesca ultramarina vasca, ni de las ca- 
racterísticas históricas de la misma, ya que existen diversos trabajos que lo han conseguido de forma ejemplar (AZKARATE, HERNÁNDEZ, NÚÑEZ, 1992: 17-32; LOEWEN, 1996; BARKHAM, 2000; GRENIER, BERNIER, STEVENS, 2007¹). Hemos preferido ofrecer una visión conjunta de los estudios realizados hasta el momento que creemos más significativos a la hora de esbozar una breve síntesis histórica.

Debemos comenzar señalando que la historiografía canadiense no ha mostrado gran interés por estudiar la presencia vasca en Canadá. Selma Huxley-Barkham es la investigadora que más ha impulsado su estudio desde que en la década de los 70 comenzara sus investigaciones en los archivos peninsulares. Su trabajo documental, esencialmente centrado en los archivos guipuzcoanos, le condujo después a estudiar las fuentes toponímicas. La conjunción de evidencias filológicas reunidas indican la existencia de una importante empresa pesquera vasca al menos desde el primer cuarto del siglo XVI en torno a la isla de Terranova, empezando por su actual capital St. Johns (San Juan de Terranova) y pasando por Placentia o Biscay Bay hasta llegar a Port-aux-Basques o Port au Choix (HUXLEYBARKHAM, 1989). La zona meridional del Labrador también figura como una zona de intensa actividad pesquera como denota la identificación de una docena de puertos con instalaciones productivas que, sumados a los de la zona oriental de Quebec y a los de Terranova, formarían parte de la red de asentamientos estacionales que los pescadores de Gipuzkoa y Bizkaia construyeron en lo que denominaban la "Gran Baya" (AZKARATE, HERNÁNDEZ, NúÑEZ, 1992: 87-91).

Aunque la principal impulsora de las intervenciones arqueológicas fue la misma Selma Huxley-Barkham, éstas fueron conducidas mayoritariamente por James Tuck y se han desarrollado con especial intensidad en la provincia de Labrador. No debemos olvidar que hasta fines de la década de los 90 del siglo XX se excavaron cinco estaciones pesqueras en Québec y una en Terranova, sin embargo, han sido los trabajos desarrollados en Labrador los que han definido el modelo de pesquerías vasco-peninsulares en Canadá (LOEWEN, 1996: 159). James Tuck, además de colaborar en los trabajos de excavación subacuática del supuesto pecio San Juan (GRENIER, BERNIER, STEVENS, 2007), también dirigió las excavaciones terrestres de Red Bay. Gracias a sus intervenciones en la Isla de Saddle se han localizando varios asentamientos temporales de filiación vasco peninsular. En estos yacimientos se documentaron hornos de fundición de grasa de ballena, talleres de carpintería para la construcción de toneles, viviendas, almacenes, embarcaderos e incluso un cementerio vasco (LOEWEN, 1996: 157).

Esta actividad terrestre fue continuada por varias campañas que se centraron en el espacio situado también en el estrecho de Belle Isle, aunque un poco más al Este, en la bahía de Chateau Bay. En la campaña inicial de prospecciones promovidas por Selma Huxley-Barkham, pero dirigidas por J. Nuñez y J. A. Hernández en 1985, se localizaron tres nuevos asentamientos temporales vasco-peninsulares, cuya existencia se ratificó mediante diferentes catas realizadas en Chateau Bay, Pleasure Harbour y Cape Charles (HERNÁNDEZ et al., 1986). En la segunda campaña, a la que se unió A. Azkarate, se excavaron en mayor extensión dos de las estaciones balleneras: Chateau Bay y Pleasure Harbour (AZKARATE, HERNÁNDEZ, NúÑEZ, 1992: 99-160). Estos trabajos permitieron exhumar evidencias materiales de hornos de fundición de grasa de ballena, talleres de carpintería y almacenes, e identificar posibles muelles y cabañas de habitación.

A pesar de la ausencia de estudios específicos al respecto en el País Vasco, pudieron constatar que el registro cerámico de los estratos asociados a esa actividad mostraba grandes similitudes con el vasco, especialmente la cerámica constructiva, muy similar a la recuperada en Gipuzkoa, Araba o Bizkaia. Al comprobar que el resto de pesquerías carecían de este rasgo constructivo, la presencia de tejas curvas de estilo árabe se convirtió en adelante en una especie de fósil-guía para denotar la presencia vasco-peninsular. La cerámica doméstica, en cambio, era aún un campo desatendido de la historiografía vasca como los propios autores reconocen (AZKARATE, HERNÁNDEZ, NÚÑEZ, 1992: 129), aunque sus esfuerzos constataron la abultada presencia de cerámica de posible procedencia francesa.

\subsection{Pesca ultramarina e identidad regional}

La lectura identitaria que la historiografía arqueológica canadiense ha realizado sobre esta empresa pesquera internacional, claramente influenciada por el muestreo documental, reduce la actividad pesquera transatlántica vasca casi por completo a las provincias más orientales del País Vasco, Gipuzkoa y Lapurdi. Una buena muestra puede encontrarse en la citada síntesis de Brad Loewen (1996: 153), quien afirma que la organización de la caza de la ballena en Labrador estaba dominada por los guipuzcoanos. Sin embargo, en estas líneas defendemos que incluso la actividad pesquera predominante de los guipuzcoanos, la pesca de la ballena, no era ni mucho menos su dominio exclusivo, ya que en ella convergían también los intereses de los comerciantes de la costa vizcaína e incluso alaveses.

No nos parece una casualidad que en 1715 fueran los Consulados y Casas de Contratación de Bilbao y Donostia los que fletaran de forma conjunta un barco, el San Nicolás, para tratar de ir a pescar a Terranova (GUIARD, 1972: 381-382). Asimismo, nos parece muy expresivo que el primer viaje conocido que partió desde el País Vasco-peninsular a Terranova en 1531 fuera fletado por un aristócrata

${ }^{1}$ Después de que escribiéramos este trabajo se ha publicado una síntesis que resulta fundamental por su exhaustividad en la revisión de los asentamientos vascos excavados en Canadá. Fue publicada en francés primero (LOEWEN, DELMAS, 2011) y en inglés después (LOEWEN, DELMAS, 2012). 


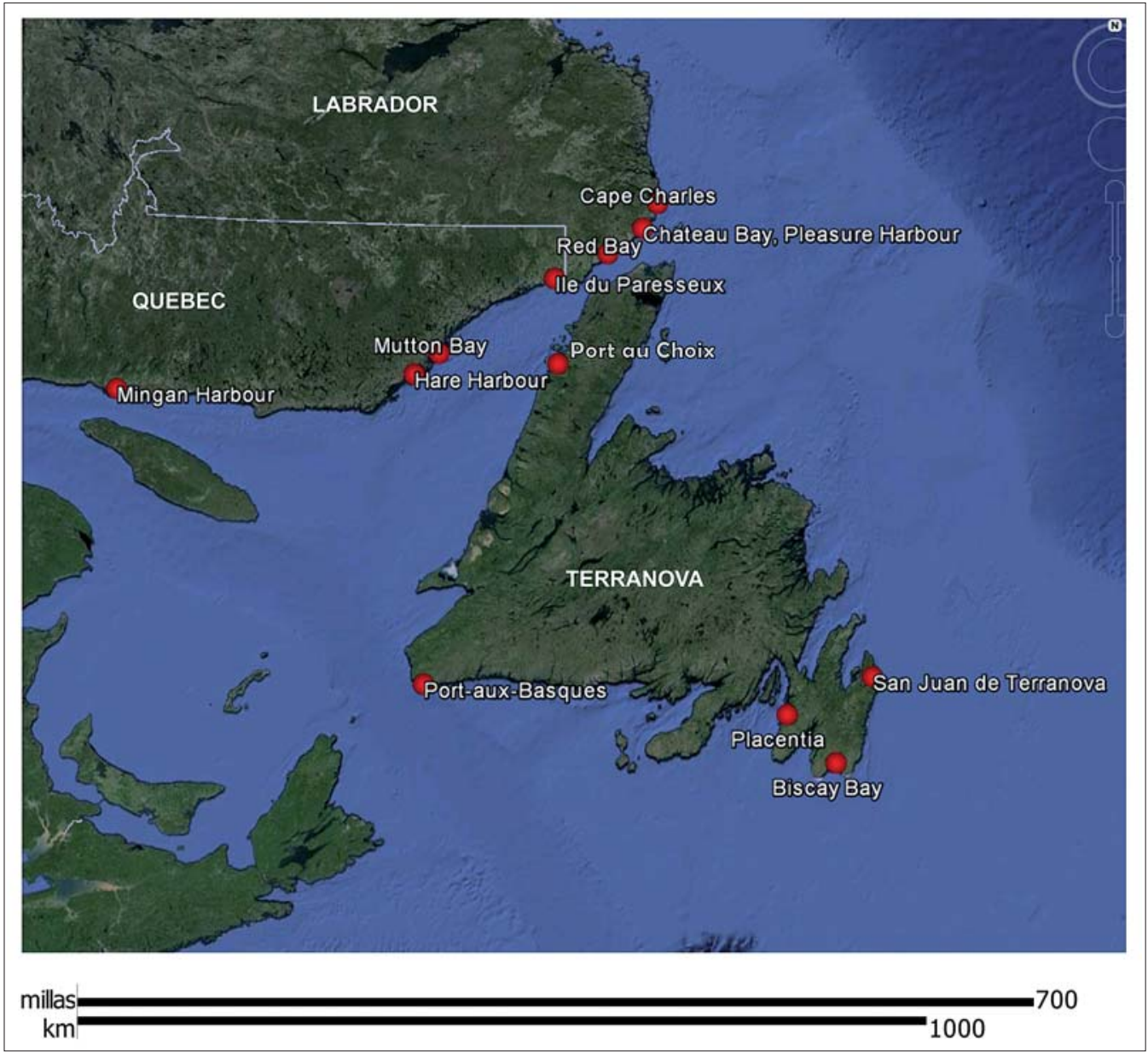

Fig. 1. Mapa de Canadá en el que se señalan los lugares mencionados en el texto. I Map of Canada with mention of the place names alluded. de Lekeitio, o que una de las primeras expediciones especializadas en el aceite de ballena fuera fletada por dos mercaderes bilbaínos (BARKHAM, 2000: 55-56). Asimismo, como nos recuerda el mismo M. Barkham, no debemos infravalorar el rol de los mercaderes y vecinos del hinterland vasco en la financiación de las expediciones pesqueras (2000: 49) y en la distribución de sus capturas (2000: 40).

Creemos, por tanto, que no se ha producido una necesaria reflexión sobre la causalidad de las fuentes. Por un lado, porque se han estudiado de forma más exhaustiva y sistemática los documentos guipuzcoanos, por sus mejores prestaciones cuantitativas y cualitativas. Por otro lado, porque las investigaciones se han centrado casi de forma exclusiva en la actividad ballenera, cuando muchas de las expediciones partieron a la pesca de bacalao, siendo muy frecuentes las empresas de naturaleza mixta. Finalmente, porque creemos que los lugares de partida de los barcos y la identidad de la empresa pesquera no pueden ni deben equipararse. Es muy significativo, a este respecto, el ejemplo que ofrece Teófilo Guiard en su Historia del Consulado y la Casa de Contratación de Bilbao, en el que describe cómo en 1564 un armador bilbaíno cede un galeón para ir a la pesca de la ballena a un capitán de Deba, quien se di- rige después a Getaria a por provisiones para el viaje y zarpa finalmente hacia Terranova (1913: 133-138).

El papel desempeñado por Bizkaia y Araba en esta empresa ha sido, en nuestra opinión, infravalorado. Aunque estos territorios quizá no fueran los más representados en la parte más visible del ciclo productivo del saín, la obtención de la materia prima y la elaboración del producto, es evidente que su papel fue importante en la inversión de capital, en la redistribución del producto elaborado y en el aprovisionamiento de los viajes; aspectos especialmente importantes para los estudios materiales del pasado y necesarios para desarrollar una interpretación global de la empresa vasca en tierras canadienses.

En la actualidad, el empleo y la consideración de nuevas fuentes documentales (PRIOTTI, 1996; BARKHAM 2000; AZPIAZU, 2008; SERNA, 2010) parecen incidir en esta dirección, y ha comenzado a igualar la balanza identitaria vasca en la pesca de ultramar. No parece una casualidad que tanto Selma Huxley-Barkham como Michael Barkham aludan de forma equitativa tanto a vizcaínos como a guipuzcoanos en la empresa pesquera canadiense desde la década de los 90 del siglo $X X$. Esta circunstancia, que también se acepta con naturalidad en la historiografía vasca más reciente (SERNA, 
2010: 46); aún no ha sido asumida por la arqueología canadiense, influenciada todavía por los primeros trabajos de Selma Huxley-Barkham que se basaron de forma casi exclusiva en la documentación escrita procedente de los archivos de Gipuzkoa.

\subsection{Nuevas yacimientos, nuevas expectativas}

Es evidente, por tanto, que la investigación arqueológica no ha avanzado en exceso desde los primeros trabajos, ni lo ha hecho su interés, tal y como dejan claro recientes síntesis sobre la arqueología histórica en Canadá, en las que a la hora de citar los trabajos arqueológicos asociados a la presencia vasca, se limitan a citar el trabajo de James Tuck y Parks Canada (DOROSZENKO, 2009: 510). Estas síntesis han omitido, de forma sorprendente, tanto la labor arqueológica vasca (AZKARATE, HERNÁNDEZ, NÚÑEZ, 1992), como las recientes aportaciones arqueológicas de William Fitzhugh, quien ha trabajado durante la última década sobre importantes evidencias materiales del poblamiento estacional vasco en Quebec. Sus intervenciones coordinadas de prospección y excavación han constatado la presencia de marineros vasco- peninsulares en la Isla de Mingan Harbour. También han documentado nuevos asentamientos de supuesto origen vasco-peninsular en el Golfo de San Lorenzo, como los yacimientos de Hare Harbour 1 en la isla de Petit-Mécatina y Havre-Boulet en Mutton Bay; o el registrado más al Este, en la zona de Blanc-Sablon, en Ile du Paresseux (FITZHUGH, 2006: 57-60).

Hare Harbour es un ejemplo de asentamiento vasco tardío. No parece que la explotación ballenera fuera muy intensa, tal y como denota la ausencia de hornos de fundición de grasa o la escasez de restos óseos de ballena, aunque los patrones de ocupación son muy similares a las pesquerías vasco peninsulares del siglo XVI, caracterizados por la presencia de estructuras con cubierta de teja, grandes recipientes de almacenaje, evidencias de trabajos de carpintería y muelles. Sin embargo, también muestra características propias, como lo son las evidencias de producción de carbón y de hierro o un registro cerámico diferente al resto de las pesquerías de filiación vasco peninsular (FITZHUGH, 2006: 62-63)². Esta circunstancia, la existencia de un asentamiento vasco tardío dedicado preferentemente a la pesca de bacalao, encaja perfectamente con la visión ofrecida por las fuentes escritas sobre la decadencia de la pesca de ballena desde fines del siglo XVI, provocada por diferentes motivos (BARKHAM, 2000: 65-72) y corrobora algunas hipótesis sobre el desarrollo de esta empresa planteadas en diferentes trabajos (AZKARATE, HERNÁNDEZ, NÚÑEZ, 1992: 88)
A la hora de establecer la cronología de las pipas de arcilla y los materiales vítreos recuperados se ha producido cierta fricción con la información procedente del registro escrito. Una cuenta de vidrio, atribuida al intercambio con los marinos vascos y fechada mediante técnicas arqueométricas, ha proporcionado una cronología que oscila entre 1680 y 1730 (FITZHUGH, 2006: 6162), una horquilla que supera ligeramente la barrera impuesta por el Tratado de Utrech al poblamiento estacional vasco-peninsular. Estas evidencias ratifican en parte la información aportada por las fuentes escritas que defienden que la presencia vasca en tierras canadienses se mantuvo hasta la primera década del siglo XVIII; aunque, a su vez, amplían su posible radio de acción temporal y dotan de mayor importancia a una actividad en supuesto declive. Así, los últimos trabajos efectuados en el yacimiento defienden que existió una clara ocupación de supuesta filiación vasco-peninsular, en forma de estación para la pesca de bacalao, entre finales del siglo XVI y principios del XVIII (FITZHUGH, FORD, 2009: 38). EI yacimiento de Petit-Mécatina se convierte así en el único asentamiento que representa, en su materialidad, la última fase de las pesquerías vascas en Canadá.

En cuanto a los contactos con las sociedades locales, en los primeros compases de explotación por parte de los pesqueros vasco peninsulares durante el siglo XVI, la zona del estrecho de Belle Isle sólo fue ocupada de forma esporádica y parcial por parte de los esquimales (FITZHUGH, 2006: 53). Sin embargo, las últimas investigaciones, han constatado la interacción entre marineros vasco peninsulares y la población esquimal, en modo de pequeñas acciones violentas (representadas sólo en las fuentes escritas), de contactos interculturales y demográficas basadas en la asistencia o servicio por parte de las poblaciones locales a los pescadores vasco peninsulares, o de intercambio tecnológico en dirección local-vasco (también documentadas en el registro arqueológico, FITZHUGH 2006: 61).

En el mismo yacimiento de la isla de Petit-Mécatina, sobre el asentamiento vasco, se han documentado incrustaciones de grasa similares a las registradas en los asentamientos locales, junto con restos de ollas que caracterizan a la cultura Dorset, extendiendo los límites de la presencia cultural de esta sociedad en época colonial hasta Quebec (FITZHUGH, 2006). Estas evidencias han sido interpretadas como un posible episodio de interacción cultural, en forma de mujer o familia esquimal al servicio de los pescadores, aunque creemos que no debería descartarse la posibilidad de que estos indicios evidencien el reaprovechamiento del asentamiento vasco por parte de los esquimales. De ser cierta la primera hipótesis, se matizaría la supuesta relación de hostilidad entre vascos y esquimales que se afirma en todas las fuentes escritas.

\footnotetext{
2 Estudios más recientes, aún sin publicar, han demostrado que estos tipos no son tan excepcionales y que se encuentran también en otros yacimientos de Labrador. La proporción de estos tipos, en cambio, sí es mayor en Petit-Mècatina, donde además existen formas diferentes (Brad Loewen, comunicación personal).
} 
La interpretación de la estratificación podría ayudar a aclarar si realmente existió un contacto directo entre pescadores y esquimales, o si, por el contrario, se trata de episodios de reaprovechamiento de las estaciones balleneras durante los meses de invierno. Las evidencias estratigráficas, aunque aún no son muy contundentes, podrían apuntar en esta última dirección, tal y como parece evidenciar el episodio de la casa esquimal construida en los primeros estratos de ocupación vasca y quemada poco después para construir sobre sus cenizas una estructura siderúrgica que, finalmente, también fue quemada (FITZHUGH, PHANEUF, 2008: 36-7).

\subsection{El papel de la cerámica}

Pero, a pesar de las repercusiones históricas de estas investigaciones para la historia canadiense, no han pasado a formar parte de la historiografía de la presencia vasca en Canadá. Por tanto, quizá sus hallazgos no han sido debidamente discutidos. Nosotros, aprovechándonos del vacío que los estudios de cerámica de época moderna nos han brindado, trataremos de hacerlo dentro de nuestra Tesis Doctoral ${ }^{3}$ y del proyecto Tecnolonial4. Porque junto a todas las estructuras descritas, también se encontraron los desechos del asentamiento, algunos bajo el agua y otros dispersos en la confusa estratificación del yacimiento (FITZHUGH, 2006: 63); y la cerámica formaba parte de esos desechos.

Uno de los aspectos que más se ha desarrollado en los estudios cerámicos de época moderna ha sido su estudio decorativo y tipológico. Por ello contamos con trabajos que, partiendo de evidencias escritas e iconográficas, han convertido la cerámica arqueológica más lujosa en indicador cronológico. Si seguimos estos criterios, podríamos considerar que en Petit-Mécatina se han recuperado varias escudillas producidas en los talleres de Muel (Zaragoza) que se fechan entre 1560 y 1610 (ALVARO, 2002: 209). Por tanto, la cerámica parece corroborar en parte la naturaleza tardía de esta pesquería, al menos su ocupación durante el siglo XVII, reforzando su condición de pesquería vasca más reciente. Y la presencia de cerámica aragonesa, muy frecuente en el registro cerámico de Época Moderna en Bizkaia y Gipuzkoa, también parece apuntalar su posible origen vasco-peninsular.

Este último planteamiento se consolida cuando consideramos que algunas de las cerámicas recuperadas en Hare Harbour se asemejan de forma asombrosa a las producidas en Bilbao entre los siglos XVI y XVII, tanto en sus características morfológicas como en las composicionales. Esta analogía a nivel compositivo se propone exclusiva- mente desde una visión arqueológica y necesariamente ha de ser contrastada mediante los análisis arqueométricos que se pretenden realizar en el marco del citado proyecto Tecnolonial (ESCRIBANO-RUIZ et al., 2010). Aunque debemos subrayar que no es un tipo cerámico muy frecuente en el registro cerámico vasco-canadiense, de confirmarse el origen propuesto, la identificación regional de los pescadores vasco-peninsulares adoptaría un nuevo rumbo, y se contaría con nuevos elementos de juicio para ahondar en la naturaleza del rol vizcaíno en esta empresa (bien sea a nivel organizativo, de aprovisionamiento o de presencia directa).

La diferencia entre el registro cerámico documentado en este yacimiento y el rescatado del pecio de Red Bay podría entenderse como efecto propio de la evolución del consumo cerámico, pero también como indicio de presencia vizcaína directa, frente a la guipuzcoana representada en el supuesto pecio San Juan. No obstante, no cabe duda de que la recuperación de este conjunto cerámico ratifica la identidad vasco-peninsular del asentamiento, si bien se presenta como una realidad diferente, que ha confundido a los investigadores, quienes incluso han llegado a dudar del origen vasco de este asentamiento pesquero (FITZHUGH, 2006: 57). Al redactar estas líneas asumimos que la presencia de productos cerámicos de origen vasco peninsular representa de una u otra forma a los pescadores vascos y no a los procedentes de otros territorios. Lo hacemos inducidos por la distribución predominantemente regional de la cerámica preindustrial vasca y porque no parece que fuera objeto de una importante comercialización a nivel internacional; pero también por la marcada tendencia al autoabastecimiento de estas expediciones pesqueras y por la ausencia de oferta de este tipo de productos en Canadá.

El estudio cerámico también permite abordar los cambios producidos por los contactos culturales en los modos de producción, especialmente en la tecnología. De la misma forma que W. Fitzhugh ha demostrado que las poblaciones esquimales influyeron en la tecnología de los europeos, como evidencia el caso del arpón metálico inspirado en modelos esquimales (2006: 58); dentro del proyecto Tecnolonial también se pretende medir si la cerámica europea, en concreto la vasco-peninsular, influyó en alguna manera en la producción cerámica local. Si bien opinamos que es un objetivo factible, no creemos que en el estudio de caso que hemos presentado se pueda constatar este proceso de influencia cultural que, al contrario, se manifiesta de forma intensa en los estudios lingüísticos. El pidgin surgido en el siglo XVI en el Golfo de San Lorenzo como consecuencia del contacto cultural entre pescadores vascos y sociedades locales es el mejor exponente a este respecto (BAKKER,

\footnotetext{
3 Titulada Genealogía del registro cerámico en época preindustrial: Araba y Bizkaia, siglos XIV al XVII, y desarrollada bajo la dirección de Agustin Azkarate. Una breve sinopsis histórica de nuestro trabajo puede encontrarse en: ESCRIBANO-RUIZ, 2012. Algunos resultados recientes pueden ser consultados en ESCRIBANO-RUIZ, 2013

${ }^{4}$ Dicho proyecto, dirigido por Jaume Buxeda i Garrigós (Universitat de Barcelona) y financiado por el Ministerio de Ciencia e Innovación (HAR2008-02834/HIST), tiene por objetivo comparar la cerámica del siglo XVI de la Península Ibérica con la recuperada en América. Para una síntesis ver: BUXEDA i GARRIGÓS, 2009. Los primeros resultados sobre la comparación entre la cerámica canadiense y la vasco-peninsular podrán ser pronto consultados en: ESCRIBANO-RUIZ et al. (e.p.).
} 


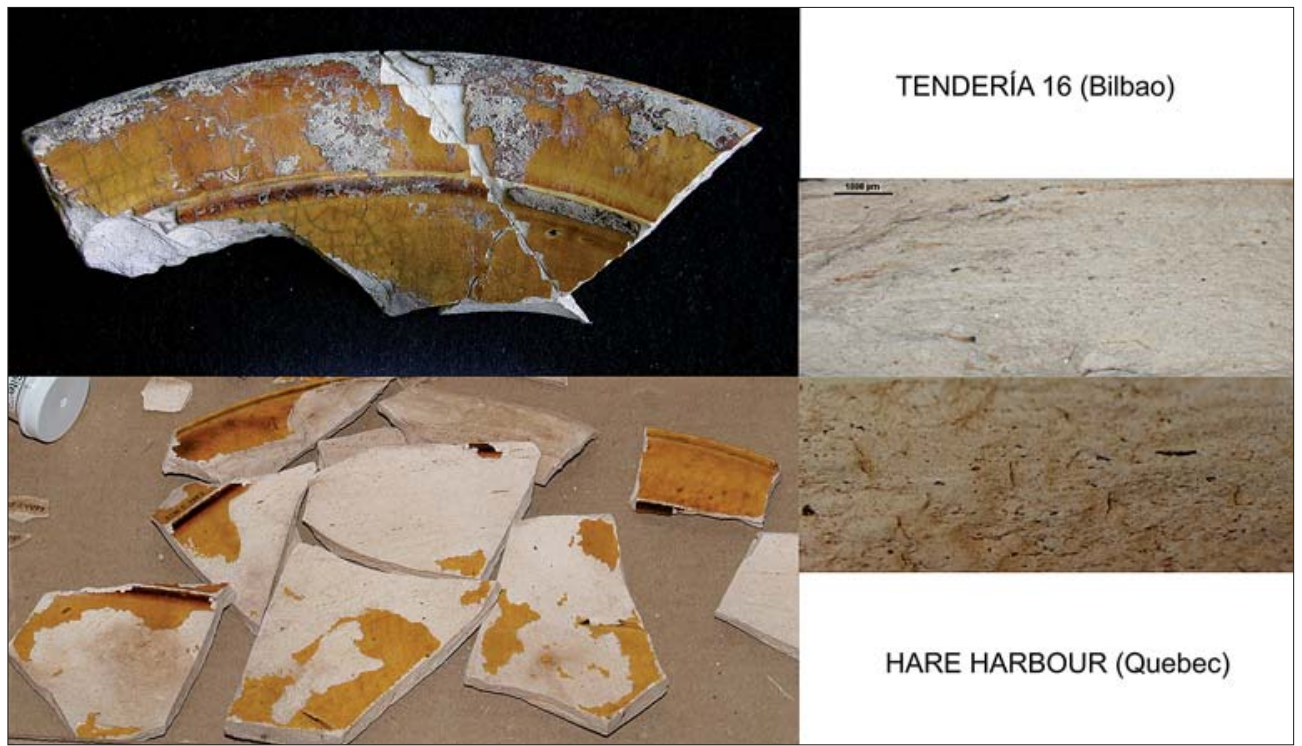

Fig. 2. Arriba: plato vidriado recuperado en Bilbao y detalle de sus pastas (Fotos: Garbiñe Dilla, Sergio Escribano-Ruiz). Abajo: plato recuperado en Petit Mécatina y detalle de sus pastas (Fotos: Anja Herzog, Sergio Escribano-Ruiz). / Top: a glazed plate recovered in Bilbao and a detail of the paste (Photos: Garbiñe Dilla, Sergio Escribano-Ruiz). Bottom: the plate recovered in Petit Mecatina Bilbao and a detail of the paste (Photos: Anja Herzog, Sergio Escribano-Ruiz).
1991). La continuidad de los modelos productivos por parte de las sociedades locales, así como la ausencia de producción europea en tierras canadienses al menos hasta el siglo XVIII, nos inducen a pensar que la influencia europea no fue tan manifiesta en la tecnología local, como lo fue en otros aspectos históricos.

\section{CONCLUSIONES}

\subsection{Sobre la diagnosis histórica documental}

Las fuentes escritas constituyen una foto de alta resolución, que ilustran muchos procesos históricos con gran nitidez. Sin embargo, generalmente responden a necesidades puntuales o a intereses concretos, por lo que no representan de la misma manera a todas las acciones del pasado ni a todos sus actores. Generan así una desigualdad informativa sobre la sociedad en la que se concibieron y sobre las sociedades con las que ésta interactuó, que resulta difícil de caracterizar y valorar por parte del investigador. En el caso que nos ocupa, por ejemplo, los documentos históricos nos informan sobre los pescadores vascos, les ponen nombres y apellidos, pero rara vez describen sus actividades con detalle o hablan de su interacción con las poblaciones locales.

\subsection{Sobre la diagnosis histórica arqueológica}

Las fuentes materiales evidencian sobre todo procesos socioeconómicos que son fuente de información estructural, que proporciona una visión más representativa a nivel social. Si bien en los siglos XVI, XVII y XVIII la escritura era un campo que excluía a la mayor parte de la sociedad, todas las escalas sociales comían y generaban desechos, o interactuaban con su paisaje urbano y natural en mayor o menor medida. Pero la gestión de sus residuos, los mecanismos de explotación medioambiental o de construcción social se nos pre- sentan muchas veces como una foto borrosa, de baja resolución, que podemos a llegar a interpretar mejor gracias al empleo crítico y simultáneo de otras evidencias. En este caso, el estudio de la cerámica de un nuevo yacimiento canadiense, nos ha servido para defender que, frente a lo que propone una historiografía canadiense auspiciada en una lectura concreta de la documentación escrita, la actividad de los marinos y comerciantes vizcaínos también fue muy importante en la empresa vasca en Canadá.

\subsection{Sobre su mutua necesidad}

Queda claro que cada evidencia proporciona una resolución temporal y dimensional muy variable; pero su correlación, atendiendo a la causalidad de cada una de ellas, es un ejercicio obligatorio si queremos obtener una comprensión multidimensional del conjunto de realidades que configuraron nuestro pasado. Pero, como hemos defendido, es un proceso que ha de ser realizado siguiendo siempre un proceso crítico, atendiendo tanto a la polifonía causa por el conjunto fuentes usadas, como a la causalidad y representatividad de cada una de ellas. En el ejemplo de la actividad pesquera vasca en tierras canadienses, esa transidisciplinaridad crítica, ha permitido aportar sólidas evidencias sobre la naturaleza y desarrollo del poblamiento estacional, así como sobre los contactos producidos entre nativos y pescadores vasco peninsulares. También ha reclamado la necesidad de investigar la identidad regional de los pescadores vascos y de ahondar en su relación con las sociedades locales a partir de las características culturales y tecnológicas de la cultura material. Creemos que el estudio presentado aporta una breve y sólida argumentación al debate global sobre el uso de fuentes históricas, abogando por un uso conjunto de fuentes escritas y materiales, guiado siempre por un proceso crítico que lo regule. 


\section{AGRADECIMIENTOS}

El contenido de este trabajo fue presentado en 2010 en el Seminario Internacional Sociedades en contacto en América Latina: prehispánico y colonial (Barcelona 10-11 de Mayo de 2010) con el título "La cerámica de procedencia arqueológica como evidencia identitaria, testando la presencia vasca en Canadá". Hemos querido mantener el contenido presentado entonces, sin actualizarlo, bajo un nuevo título. Agradecemos especialmente la ayuda y colaboración de una de las organizadoras del seminario, Natalia Moragas Segura (Universitat de Barcelona). Debemos agradecer al Campus de Álava de la Universidad del País Vasco (UPV/EHU) y a la Diputación Foral de Álava la financiación aportada, mediante la convocatoria Tesi ikerkuntza 2010, para asistir a este evento.

La comunicación mantenida con Brad Loewen ha sido la promotora de este trabajo, y sus comentarios han supuesto revisar algunas de nuestras palabras. Por ello, queremos mostrarle esta muestra de agradecimiento. Igualmente, queremos agradecer a William Fitzhugh y Anja Herzog, el acceso y uso de la información relativa al registro cerámico de Hare Harbour 1. El trabajo se ha desarrollado dentro del proyecto Impacto tecnológico en el Nuevo Mundo colonial. Aculturación en arqueología y arqueometría cerámica (TECNOLONIAL), financiado por el Ministerio de Ciencia e Innovación (HAR2008-02834), cuyo investigador es principal Jaume Buxeda i Garrigós. Julio Nuñez y Agustín Azkarate también han proporcionado una colaboración directa y/o indirecta en la redacción de esta breve reflexión.

\section{BIBLIOGRAFÍA}

ALVARO, M. I.

2002 Cerámica aragonesa, vol. II. Ibercaja. Zaragoza.

\section{AMORES, F.}

1997 La arqueología postmedieval en España. Panorama y perspectivas. Archeologia Postmedievale 1, 51-67.

AZKARATE, A.; HERNÁNDEZ, J. A. \& NÚÑEZ, J.

1992 Balleneros vascos del siglo XVI. Gobierno Vasco. VitoriaGasteiz.

AZPIAZU, J. A

2008 La empresa vasca de Terranova. Entre el mito y la realidad. Ttartalo. Donostia.

BAKKER, P.

1991 Un pidgin vasco y amerindio, en BAKKER, P., BILBAO, G., DEEN N. G. H. \& HUALDE, J.I. Basque pidgins in Iceland and Canada. 134-165. Anejos del Anuario del Seminario de Filología Vasca Julio de Urquijo. Gipuzkoako Foru Aldundia. Donostia.
BARKER, D. \& MAJEWSKI, T.

2006 Ceramic studies in historical archaeology, en HICKS, D. \& BEAUDRY, M.C. The Cambridge Companion to Historical Archaeology. 205-231. Cambridge University Press. Cambridge.

\section{BARKHAM, M.}

2000 La industria pesquera en el País Vasco peninsular al principio de la Edad Moderna: ¿una edad de oro? Itsas Memoria. Cuadernos de estudios marítimos del País Vasco 3, 29-75.

\section{BUXEDA i GARRIGÓS, J.}

2009 Impacte tecnològic en el nou món colonial. Aculturació en arqueologia i arqueometria ceràmica (TECNOLONIAL). Quarhis 5, 222-224.

\section{DOROSZENKO, D.}

2009 Exploration, exploitation, expansion and settlement: historical archaeology in Canada", en MAJEWSKI, T. \& GAIMSTER, D. International Handbook of Historical Archaeology. 507-523. Springer. New York.

ESCRIBANO-RUIZ, S.

2012 El registro cerámico del País Vasco, Álava y Bizkaia, siglos XIV al XVII. Retrospectiva histórica, en CASTRO CORREA, A., GÓMEZ CASTRO, D., GONZÁLEZ GERMAIN, G., STARCZEWSKA, K., OLLER GUZMÁN, J., PUY MAESO, A., RIERA VARGAS, R. \& VILLAGRA HIDALGO, N. Estudiar el pasado: aspectos metodológicos de la investigación en ciencias de la Antigüedad y de la Edad Media. 231-236. BAR International Series 2370. Oxford.

2013 Los antecedentes de la cerámica popular vasca. Consideraciones desde el consumo cerámico de Durango y Vitoria-Gasteiz (siglos XIV y XVII), en GOMEZ DE SEGURA, B. Siglos de Alfarería en Ollerías. 34-61. Ayuntamiento de Legutio - Museo de Alfarería Vasca. Legutio.

ESCRIBANO-RUIZ, S.; BUXEDA i GARRIGÓS, J.; MADRID i FERNÁNDEZ, M.; NUÑEZ MARCÉN, J. \& AZKARATE GARAI-OLAUN, A.

2010 Tracing the Basque presence in Eastern Canada during the 16th and 17th centuries through pottery remains: the example of lead-glazed pottery produced in Bilbo. Póster presentado en Exploring New World Transitions: from Seasonal Presence to Permanent Settlement, Society for Post-Medieval Archaeology Conference, 16-20 June, St John's (Newfoundland).

ESCRIBANO-RUIZ, S., LOEWEN, B., AZKARATE, A., BARRACHINA C. P., NUÑEZ. J. \& MONETTE, Y.

(e.p.) Basque Fishing Crews' Pottery in Canada. A Transatlantic Evaluation of Ceramic Remains Left by an Early Modern Global Enterprise, en BUXEDA i GARRIGÓS, J., MADRID i FERNÁNDEZ, M. \& IÑAÑEZ, J. G. Global Pottery 1. Historical Archaeology and Archaeometry for Societies in Contact. BAR International Series. Oxford.

\section{FITZHUGH, W.}

2006 Cultures, borders, and basques: archaeological surveys on Quebec's lower north shore, en RANKIN, L.\& RAMSDEN, P. From the Arctic to Avalon. Papers in Honour of Jim Tuck. 53-70. BAR International Series 1507. Oxford. 
FITZHUGH, W. \& FORD, B.

2009 The Gateways Project 2008. Land and underwater excavations at Hare Harbour, Mécatina. Arctic Studies Center, Smithsonian Institution. Washington DC.

FITZHUGH, W. \& PHANEUF, E.

2008 The Gateways Project 2007. Land and underwater excavations at Hare Harbour, Mécatina. Arctic Studies Center Smithsonian Institution. Washington DC.

FUNARI, P. P. A.; HALL, M. \& JONES S. (Eds.)

1999 Historical Archaeology. Back from the Age. Routledge. London.

GRENIER, R.; BERNIER, M.A. \& STEVENS, W. (Eds.)

2007 The underwater archaeology of Red Bay: Basque ship-building and whaling in the $16^{\text {h }}$ century. Parks Canada. Ottawa.

GUIARD, T.

1972 Historia del Consulado y la Casa de Contratación de Bilbao, vol II. Editorial la Gran Enciclopedia Vasca. Bilbao.

HERNÁNDEZ, J. A.; BIENES, J.; NUÑEZ, J. \& ZUMALDE, I.

1986 Basque expedition to Labrador. Archaeology in Newfoundland and Labrador 1985, 81-98.

HICKS, D. \& BEAUDRY, M. C. (Eds.)

2006 The Cambridge Companion to Historical Archaeology. Cambridge University Press. Cambridge.

\section{HUXLEY-BARKHAM, S.}

1989 The Basque Coast of Newfoundland. The Great Northern Peninsula Development Corporation. St. Johns.

JOYCE, $\mathrm{R}$

2006 Writing historical archaeology, en HICKS D. \& BEAUDRY M.C. The Cambridge Companion to Historical Archaeology. 48-65. Cambridge University Press. Cambridge.
LOEWEN, B.

1996 El estudio de la historia marítima vasca en Canadá. Itsas Memoria. Cuadernos de estudios marítimos del País Vasco 1, 151-166.

\section{LOEWEN, B. \& DELMAS, V.}

2011 Les occupations basques dans le golfe du Saint-Laurent, 1530-1760. Périodisation, répartition géographique et culture matérielle. Archéologiques 24, 23-55.

2012 The Basques in the Gulf of St. Lawrence and Adjacent Shores. Canadian Journal of Archaeology 36, 213-266.

MAJEWSKI, T. \& GAIMSTER, D. (Eds).

2009 International Handbook of Historical Archaeology. Springer. New York.

MAJEWSKI, T. \& SCHIFFER, M. B.

2009 Beyond consumption: toward an archaeology of consumerism, en MAJEWSKI, T. \& GAIMSTER, D. International Handbook of Historical Archaeology. 191-207. Springer. New York.

ORSER, C.E. Jr. (Ed.).

2002 Encyclopedia of Historical Archaeology. Routledge. London.

PRIOTTI, J.P.

1996 Los Echávarri: mercaderes bilbaínos del Siglo de Oro. Diputación Foral de Bizkaia. Bilbao.

SERNA, M.

2010 Los viajes pesquero-comerciales de guipuzcoanos y vizcaínos a Terranova (1530-1808): régimen jurídico. Marcial Pons, IVAP. Madrid.

WHITE, C. L. \& BEAUDRY, M. C.

2009 Artifacts and personal identity, en en MAJEWSKI, T. \& GAIMSTER, D. International Handbook of Historical Archaeology. 209-225. Springer: New York.

WILKIE, L. A.

2006 Documentary archaeology, HICKS D. \& BEAUDRY M.C. The Cambridge Companion to Historical Archaeology. 1333. Cambridge University Press. Cambridge. 
